\title{
OPTIMISATION OF THE DEPLOYMENT SEQUENCE OF 2 DOF SYSTEMS
}

\author{
G.E. FENCI \& N.G.R. CURRIE \\ Directorate of Civil Engineering, University of Salford, Salford, United Kingdom.
}

\begin{abstract}
The methodology for the analysis of deployable structures with 2 degrees of freedom (DoF) and optimisation of the deployment sequence is proposed. A parametrically controlled geometry, based on the design of biomimetic deployable structures, is systematically cycled through all available combinations of deployment and analysed for the full range of available motion established by the two DoFs. In other words, structural analysis is carried out for all potential configurations for the $2 \mathrm{DoFs}$, which act independently from one another. The results are, then, automatically post-processed to give contour plots showing the change in performance criteria such as the force or moment that develops in the structure during deployment. Knowing that the structure needs to deploy from the fully folded to the fully unfolded state, the generation of convex hull profiles allows the selection of the optimum path to reach the fully deployed state based on whatever the governing criteria is deemed to be, such as maximum deployment force, deflection, weight of structure, or in service stresses.

Keywords: brute force, convex hull, deployable structures, deployment sequence, optimisation.
\end{abstract}

\section{INTRODUCTION}

Typically starting from a compact, folded configuration, deployable structures have the capacity to expand, through a series of controlled motions [1], to a larger, more open structure. Equally, many deployable structures can reverse the process or retract [2]. The classification table by Hanaor and Levy [3] illustrates the variety of deployable structures that include scissor-hinged structures $[4,5]$; folded plates, often linked to the science of origami $[6,7]$; tensegrities, based on the separation of the tensile forces from compression ones $[8,9]$; and membrane structures, such as boundary tension membranes and pneumatic structures $[10,11]$.

Traditionally, structural engineering is concerned with the performance of structures in their final in-service form or the temporary works associated with construction. Deployable structures need no temporary works within their deployment, but require complex (often non-linear) analysis to understand both their repeatable geometrical movements, their deployment forces, and material stresses [12]. This frequently results in difficulty in finding a single working solution, let alone optimising the performance of the structure through its available deployment range.

When optimising deployable structures a key objective is minimum weight, which means the corresponding reduction of the magnitude of the actuation required for deployment. You [13] optimised the geometry of a triangular pantographic mast via a sensitivity analysis based on the force method, while Gantes, Georgiou and Koumousis [14] used genetic algorithms to optimise the material and section properties of a snap-though deployable structure. Other optimisation of rigid link deployables includes attempts to employ genetic algorithms and ant colony optimisation for sizing optimisation $[15,16]$ and a nested approach that iterates between shape and sizing optimisation, using stochastic searches and gradient-based algorithms [17]. Finally, Dai and Guan [18] employed sequential quadratic programming to explore the design space and optimise a pantographic structure to find the minimum mass solution. Fest, Shea, Domer and Smith [19] applied a newly developed stochastic search 
algorithm to identify appropriate control commands for a reusable tensegrity structure and a method for determining the optimised shapes of cover plates for circular retractable roofs was suggested by Buhl, Jensen and Pellegrino [20]. The objective of the plates' optimisation was to minimise overlap throughout deployment as well as the gap between the plates when the roof was closed and to maximize the area of the opening, rather than focusing the structure's overall weight. A method for the optimisation of both self-weight and overall displacement for a movable telescope support was proposed by Ortega and Robles [21].

\section{METHODOLOGY}

The focus of this research was on the optimisation of deployable structures with 2 degrees of freedom (DoF). Due to the structures having two distinct ranges of motion, which act independently from one another, the potential deployment sequences are close to infinite. The optimisation process aims at finding the optimum deployment sequence that will minimise the stresses and control deflections within the structure during its motion through the deployment configurations.

First of all, the variable geometry had to be modelled in a manner that would easily allow modifying the parameters governing the DoF through parametric definition. The first geometry taken into consideration was that of a deployable canopy formerly analysed [22].

The canopy is a biomimetically inspired deployable structure based on the blooming of a swirl flower with three DoF. For the purpose of this research the third degree of freedom was limited in order to provide a 2 DoF system to establish a methodology, see Fig. 1. While, previously, the structure's parametric geometry had been determined via rotation and translation about fixed points via geometric matrix operations, this time it was developed by creating an algorithmic sequence in Grasshopper, a visual programming language by Rutten [23]. The structure was defined by the following variable parameters: bottom radius, ratio of top to bottom radius, number of struts, strut length, elevation of the bottom plate, and rotation of the bottom plate - these last two parameters being the DoF (Fig. 2).

The DoF were represented each by their own slider; varying one or both of them changed the structure's configuration and, consequently, the forces and stresses developing along the struts.

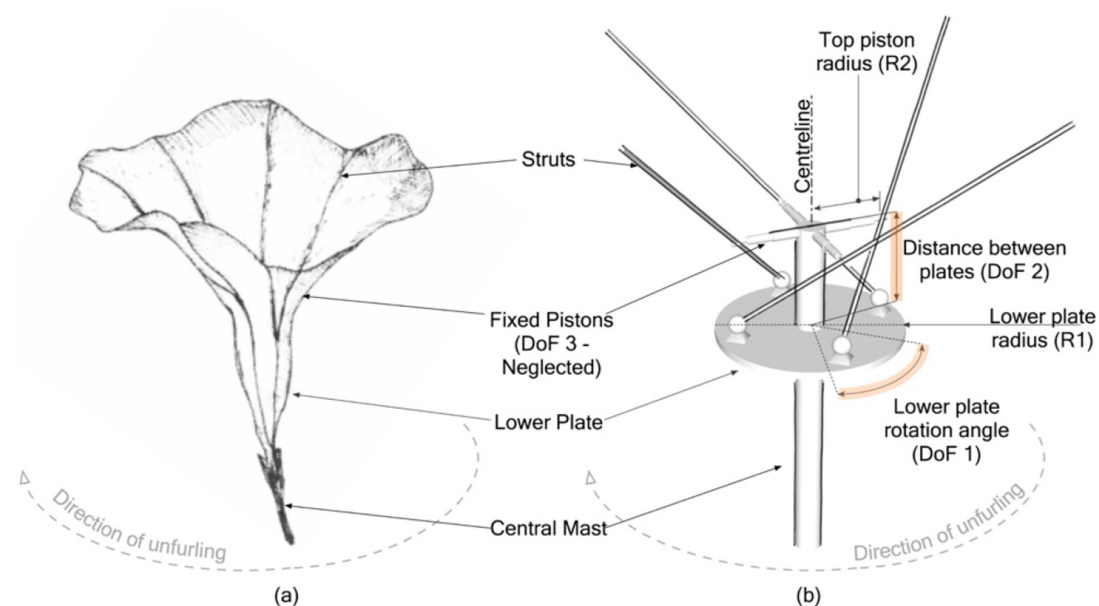

(a)

(b)

Figure 1: Biomimetically inspired deployable canopy. 
Once the deployable canopy had been modelled, structural analysis was carried out by applying material properties via Karamba [24], a Grasshopper plugin that provides an interactive and parametric finite element program for shell and beam structures (Fig. 3). Before proceeding with the optimisation, the results of the structural analysis were validated by modelling the same structure in Autodesk Robot Structural Analysis [25] and comparing the results.

As the structure starts being fully folded and progresses to its fully deployed position, the sliders range from zero to their maximum value, representing the maximum rotation or elevation allowed to reach the unfolded state. In order to limit the number of cases analysed, the

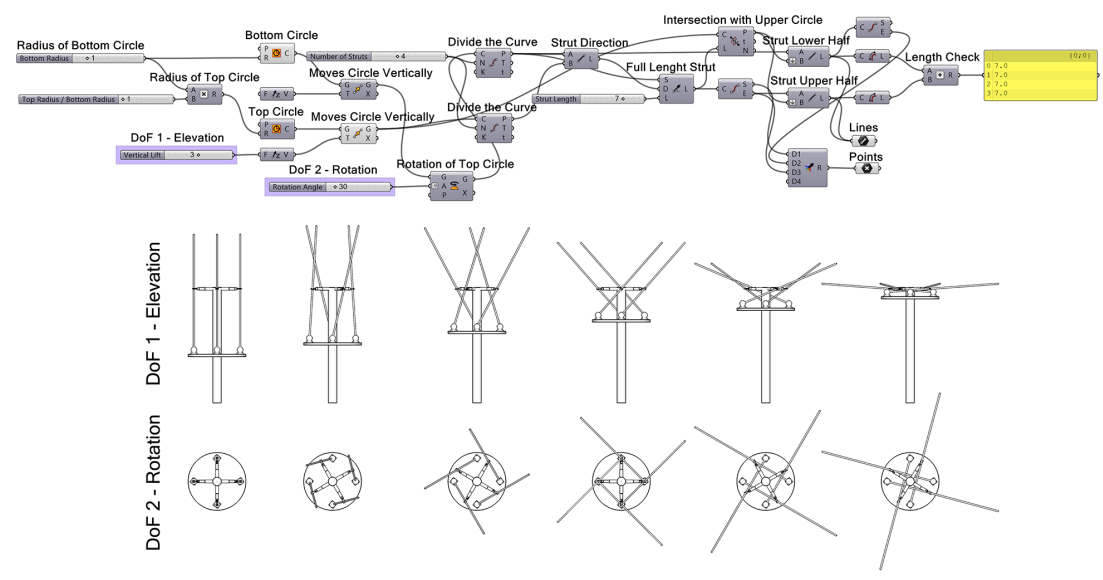

Figure 2: Algorithm for deployable canopy.

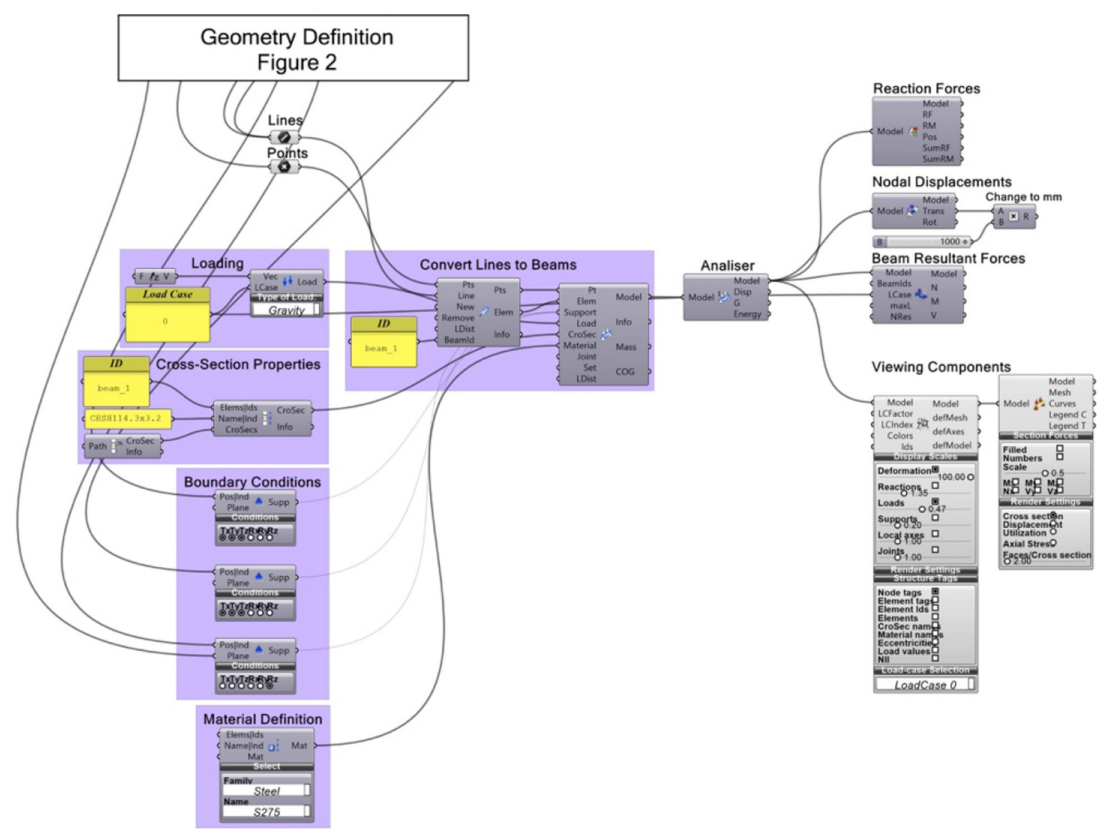

Figure 3: Analysis algorithm using Karamba. 
sliders were set to assume a limited number of values. Five elevation heights and five rotation angles were defined, making the potential combinations equal to 25 , although more refined combinations were equally achievable at the expense of processing time.

If one were to manually manipulate the sliders to allow Karamba to analyse every deployment configuration it would take a considerable amount of time, hence, a plugin that would automatically generate all the potential combinations was required. Such characteristic was found in TT Toolbox, a Grasshopper plugin featuring a range of tools developed by the Core Studio at Thornton Tomasetti (former Advanced Computational Modelling Group). One of the tools, called the Brute Force component, offers another means for running optimisation routines in Grasshopper, in the sense that, instead of finding the optimal combination of sliders, it simply solves all possible combinations of sliders that are connected to its input. This component works in combination with Galapagos Listener, another tool belonging to the same plugin [26].

The setup in Fig. 4 allows running through all of the possible combinations of sliders " $u$ " and " $v$ " while recording the corresponding numeric fitness value. The Listener component stores the values after the Brute Force component has finished solving, so that they may be used further in the algorithm.

Due to the importance of scale when post-processing the results, the deployment stages had to be the same for both of the degree of freedom sliders. The table in Fig. 5 shows the amount of elevation and rotation relative to the percentage of deployment, where $0 \%$ indicates fully folded and $100 \%$ fully deployed. Once the algorithm was connected to the Karamba components for the analysis and the Brute Force component was run, each

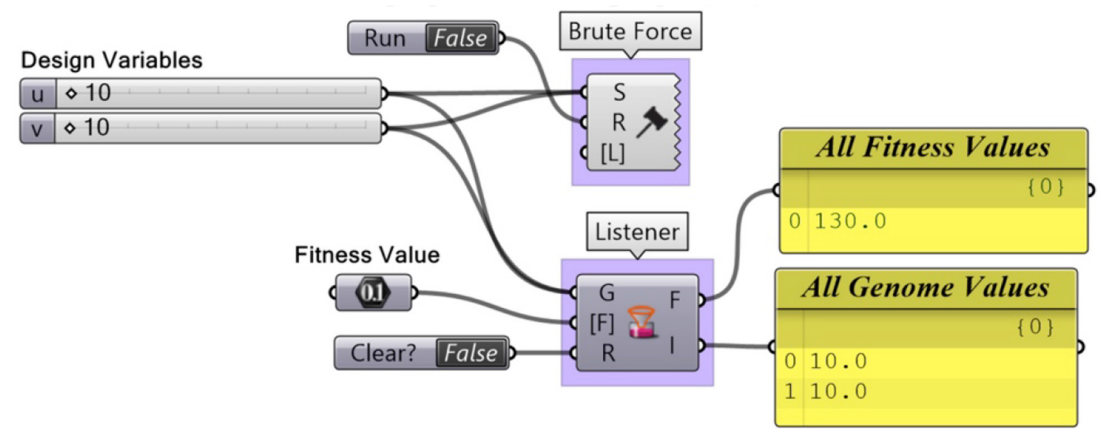

Figure 4: Brute force Grasshopper setup with Galapagos Listener component.

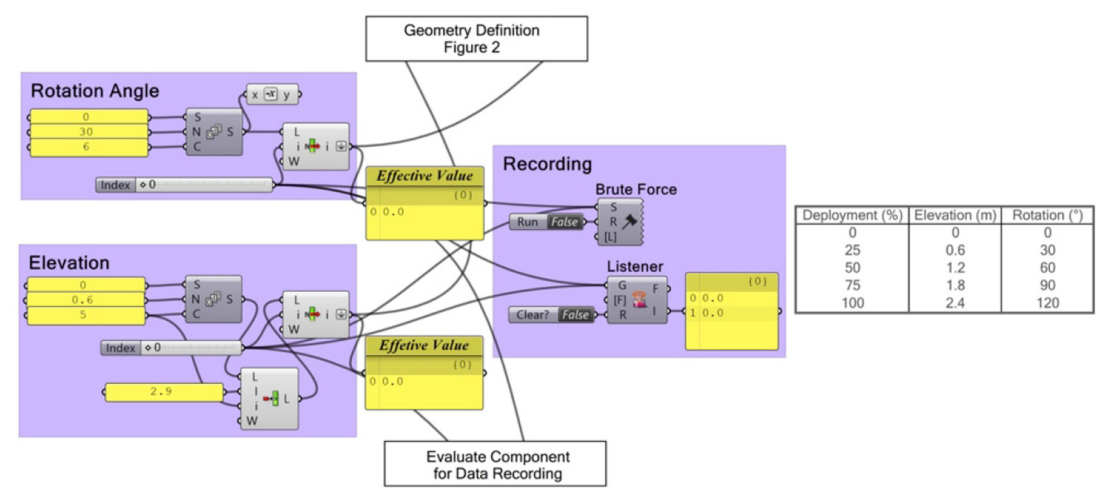

Figure 5: Brute force setup for deployable canopy and index for DoFs. 
deployment stage, determined by the variation of the sliders, was automatically structurally analysed.

All the results were combined into a single output via an Evaluate component and recorded by a Data Recording component linked with the panel showing the complete output. The content of the panel was streamed to a comma separated values file (csv), which could be imported and used by other software using common protocols.

The results were post-processed in Wolfram Mathematica (Version 10) [27], a symbolic mathematical computational program. Through a series of list manipulations, contour plots showing the variation of each force, stress, and deflection developed in the structure for all combinations of the 2 DoFs were created. The reason for highlighting scale when brute forcing the sliders was because, in order to create accurate contour plots, it was necessary for the number of values and the values themselves for each axis to be the same, to avoid running into scaling errors. The axes were labelled with the percentage of deployment for each DoF. Looking at the contour plot for the axial forces acting on the plate and that for the strut deflection in Fig. 6, if the axes had a different number of intervals, or those intervals were represented by different values the overall scale of the plots would be incompatible.

The contour plots in Fig. 6 represent two different scenarios showing where the hotspots developed (red area) and how the intensity of the force/deflection varied throughout the combinations of rotation and elevation. The plots were used to find the path from $(0,0)$ fully folded to $(100,100)$ fully deployed, which caused the least amount of axial force and displacement in the structure. Looking at the contour plot for axial force (case 1), initially, the outermost contour was selected via a short script. The thought process that followed the contour selection is best understood when looking at Fig. 7, which shows the results outputted from the Mathematica code for the axial force condition.

The first consideration made was that the deployment path causing the least axial force would be enclosed in the purple area where the minimum values were located, while the shortest path would be a diagonal connecting $(0,0)$ and $(100,100)$, thus, the optimum path would result as a compromise between the two. The shortest path translates into a quicker deployment sequence as it implies the DoF acting simultaneously, rather than them reaching full deployment during separate stages. To optimise the path, an initial vector starting from $(0,0)$ had to be created that intersected with the selected contour and made the furthest
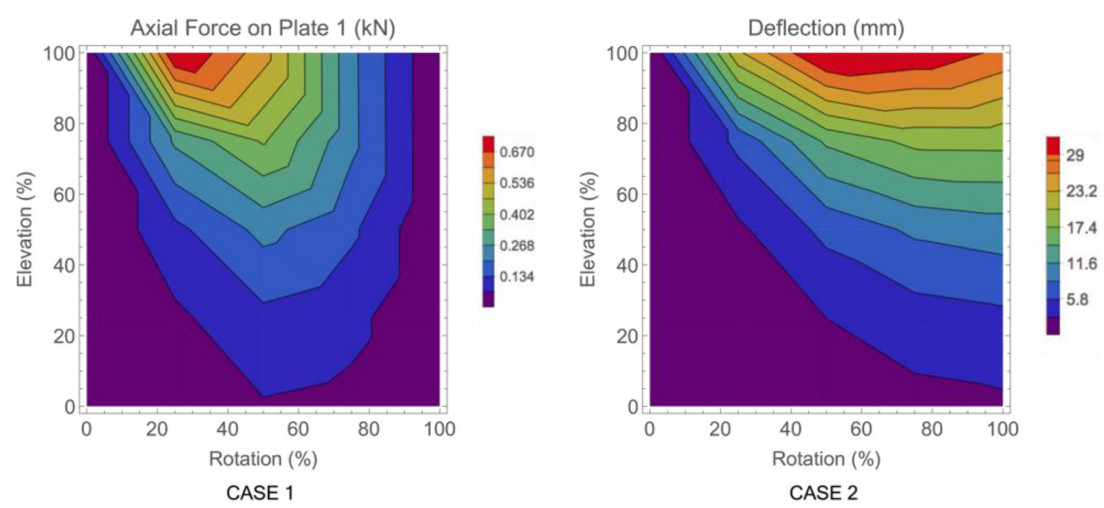

Figure 6: Example of contour plot. 

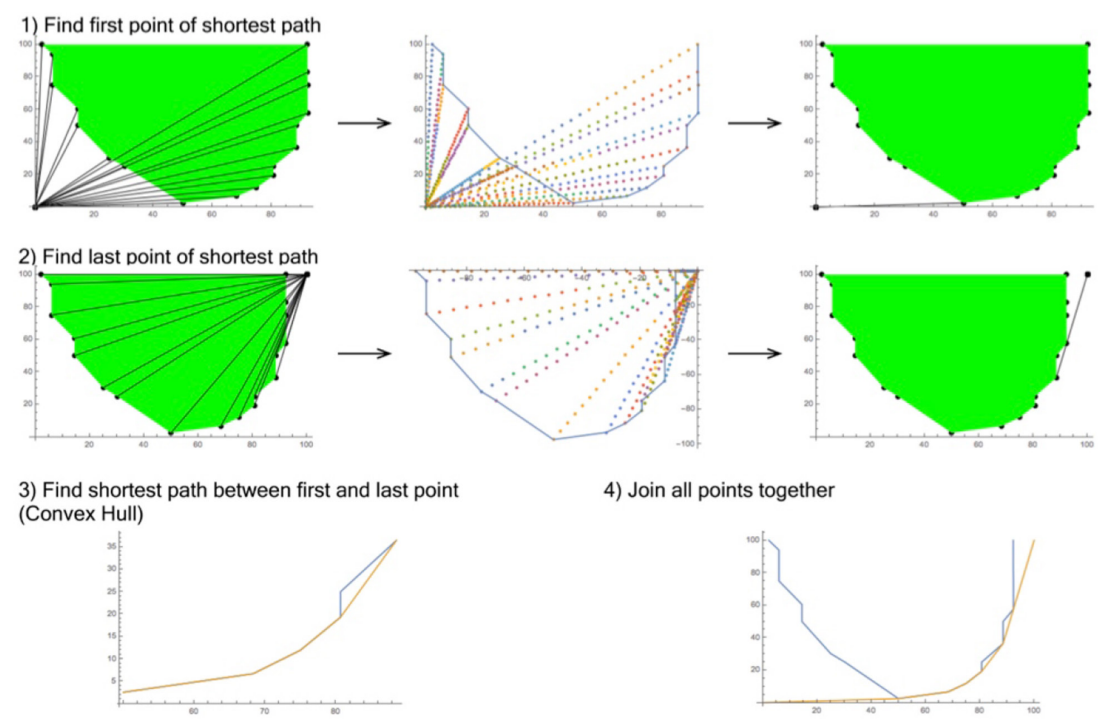

4) Join all points together

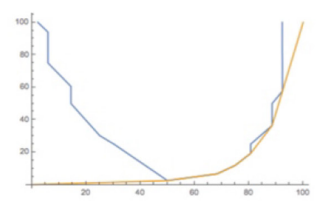

Figure 7: Process of finding the shortest path.

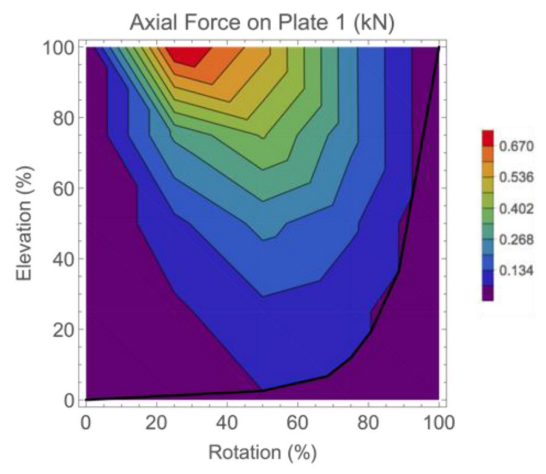

CASE 1

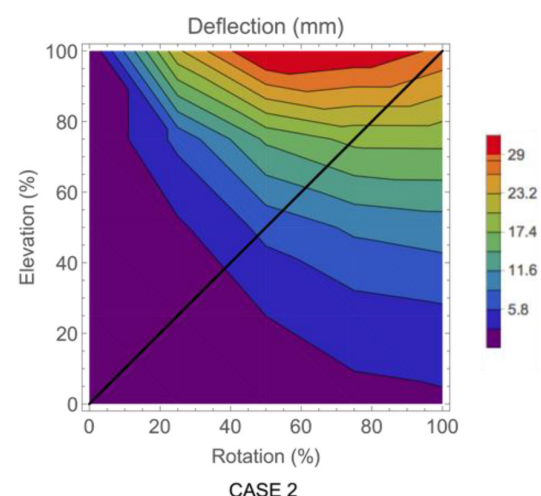

CASE 2

Figure 8: Optimum deployment paths.

distance along the contour in the x-axis without actually crossing the contour, essentially creating an approach vector. To verify if an approach vector crossed the selected contour a Boolean check based on the winding number of points along the approach vector was performed. The winding number verification outputted "False" for points outside the contour and "True" for the ones inside and only the lines where all points lied outside the contour area were selected as valid solutions. The last item on the list was picked and connected to $(0,0)$.

The same process, only reversed, was followed to find the last point of the contour to connect to the fully deployed state $(100,100)$ without crossing the selected contour, this being the exit vector. Next, a line connecting the approach vector and exit vector that followed the contour points had to be determined. This line could not cross the contour and had to be the shortest path. Thus, instead of following the contour profile, which would be an obvious but 
not optimal path, the path was shortened by calculating its convex hull, i.e. the shortest convex set containing those contour points. Finally, all points were connected to one another and the optimum path defined.

The routine was written to account for various scenarios at the same time, such as the one resulting from the deflection of the struts (case 2), see Fig. 8. In this case it was impossible to keep the path within the purple area, as it necessarily needed to cross all contours except for the red one. Thus, the routine neglected the middle convex hull process and the criteria of choosing the quickest path governed by outputting a diagonal connecting the two extremes.

The optimisation process described was carried out for all the lists imported from the csv file, i.e. for all the values of forces, stresses, and displacement outputted by the Karamba analysis.

\section{DISCUSSION}

The methodology proposed for the optimisation of the deployment sequence of a structure characterised by 2 DoFs is summarised in Fig. 9.

First, the geometry is defined and the DoF are assigned a slider. All the potential combinations of the DoF are, then, fed to the structural analysis algorithm, the output of which is then post-processed in to find the optimum deployment path.

Compared to methodologies previously adopted, the processing time was significantly decreased due to brute forcing of all the potential combinations of the DoF for the structural analysis to solve a vast array of deployment scenarios instead of having to manually change the structure's deployment stages.

Once all the data obtained from the structural analysis were streamed to a csv file, this was summoned into a Mathematica workbook, ready for post-processing. The code automatically separated each list of the data, recognising which values indicated the percentages of deployment and which the values for forces, stresses, and displacements. Then, the contour plots were drawn.

This optimisation process is innovative due to its flexibility, as it can be used for any problem with 2 variables. Specifically with regards to deployable structures, independently of the structure's geometry or its deployment mechanism, as long as there are two parameters

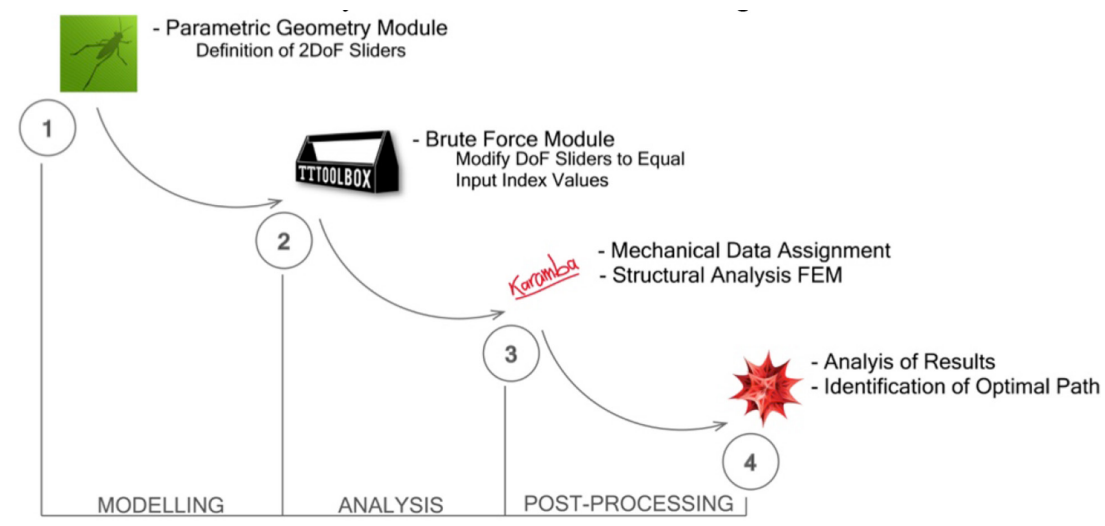

Figure 9: Summary of optimisation process. 


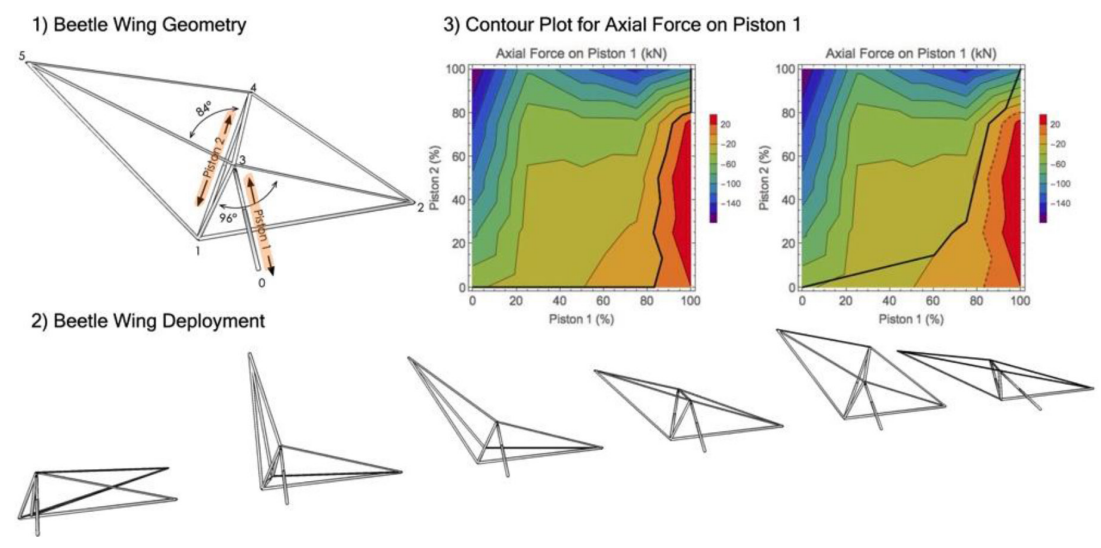

Figure 10: Methodology applied to a different deployable structure.

representing the degrees of motion, the deployment optimisation can be carried out for any criteria, such as energy necessary for deployment, overall weight of the structure, etc.

Let us consider a different biomimetic deployable structure, this time inspired by the unfolding of a beetle wing and the Miura Ori origami fold [28]. The structure is pinned so no moments develop along the struts apart from those due to self-weight. Once again, there are 2 DoFs represented by two pistons, which determine the wing's deployment. Figure 10 shows the structures' geometry and one of the potential deployment sequences as well as the contour plot for the axial force developing in piston 1. Initially, the piston's length is at a minimum, meaning that its capacity to withstand the load, will be greater, while, as the deployment progresses, its length increases and the forces acting upon it should, hence, be contained.

The dotted line in the contour plots in Fig. 10 represents a change in sign: to the left of the line the piston is in compression and in tension on the right-hand side. In this case, the advantage of using the contour plots for determining the deployment sequence is that the designer can choose to avoid tension completely and contain the deployment path on the left-hand side of the 0 contour or reach the 0 axial force contour and follow its profile for as long as possible to reach the fully deployed stage, thus, minimising the compression/tension force the piston has to withstand (see plots in Fig. 10).

Finally, the number of deployment stages analysed for the deployable canopies was limited to 25 , however, accuracy could be increased if more stages were run through the analysis and if more contour lines were plotted.

\section{CONCLUSION}

This research project has considered the optimisation of the deployment sequence of deployable structures with 2 DoFs. The structures on which the optimisation was carried out are biomimetically inspired canopies based on the imitation of the blooming of a flower and the unfolding of a beetle wing.

The optimisation methodology, first, requires the structure to be parametrically defined with two distinct DoF. Brute forcing the variable parameters representing the DoFs allows for the structural analysis to run a considerable amount of analysis representing potential deployment stages given by different combinations of the DoFs, providing a complete dataset. The output is, then, transferred to a mathematical computational program and post- 
processed to allow scenarios to be explored and for the designer to gain a feel for the overall structural behaviour.

This stage is where the optimisation actually takes place. A custom script was written to process the structural analysis results and use them to create contour plots illustrating the distribution of forces, stresses and displacements for all the deployment stages analysed. The plots highlighted the hot-spots where the maxima occurred and the areas where the forces reached their minimum value. By containing the deployment paths within these areas the deployment sequence can be optimised to reduce either the weight of the structure or deployment force. In addition to limiting the deployment path within the area representing the deployment stages causing the least stress for the structure, the shortest path was sought to enable the structure to reach the fully deployed state quicker.

By applying the methodology to two distinct structures, it was demonstrated how the optimisation process is versatile and may be applied to any deployable structure with 2 DoFs. However, there are a various governing criteria that could result from applying this methodology and engineering judgement is necessary to select an appropriate contour path.

Future work includes applying the proposed methodology to an array of deployable structure with 2 DoFs to establish potential trends and to further improve the coding to account for more contour plots scenarios. Finally, the methodology will be advanced to consider structures with an added degree of freedom resulting in the creation of 3D intensity graphs.

\section{REFERENCES}

[1] Tibert, G., Deployable Tensegrity Structures for Space Applications, Department of Mechanics, Royal Institute of Technology, 2002.

[2] Pellegrino, S., Deployable Structures, Wien: Springer, 2001.

[3] Hanaor, A. \& Levy, R., Evaluation of deployable structures for space enclosures. International Journal of Space Structures, 16(4), pp. 211-229, 2001. http://dx.doi.org/10.1260/026635101760832172

[4] Escrig, F. \& Valcarcel, J.P., Geometry of expandable space bar structures. International Journal of Space Structures, 8(1/2), pp. 71-84, 1993.

[5] Piñero, E.P., Three dimensional reticular structure. U.S. Patent No. 3,185,164, 1965.

[6] Schenk, M. \& Guest, S.D., Origami folding: A structural engineering approach. Origami, 5, pp. 291-304, 2011. http://dx.doi.org/10.1201/b10971-27

[7] Tachi, T., Geometric considerations for the design of rigid origami structures. Proceedings of the International Association for Shell and Spatial Structures (IASS) Symposium 2010, Shanghai, pp. 458-460, 2010.

[8] Fuller, R.B., Tensile-integrity structures," U.S. Patent No. 3,063,521, 1962.

[9] Motro, R., Tensegrity systems: the state of the art. International Journal of Space Structures, 7(2), pp. 75-83, 1992, 1992.

[10] Otto, F., Convertible roofs. Institut for Leightweight Structures, Univ. Stuttgart, IL5, 1971.

[11] McLean, W. \& Silver, P., Air Structures, Laurence King Publishing, 2015.

[12] Gantes, C.J., Deployable Structures: Analysis and Design, Southampton: WIT Press, 2001.

[13] You, Z., Sensitivity analysis based on the force method for deployable cable-stiffened structures. Engineering Optimization, 29(1-4), pp. 429-441, 1997.

http://dx.doi.org/10.1080/03052159708941006 
[14] Gantes, C., Georgiou, P. \& Koumousis, V., Optimum design of deployable structures using genetic algorithms. WIT Transactions on the Built Environment, 38, 1998.

[15] Kaveh, A. \& Shojaee, S., Discrete-sizing optimal design of scissor-link foldable structures using genetic algorithm. Asian Journal of Civil Engineering (building and housing), 4(2-4), pp. 115-133, 2003.

http://dx.doi.org/10.1111/j.1467-8667.2006.00470.x

[16] Kaveh, A. \& Shojaee, S., Optimal design of scissor -link foldable structures using ant colony optimization algorithm. Computer-Aided Civil and Infrastructure Engineering, 22(1), pp. 56-64, 2007.

[17] Thrall, A.P., Zhu, M., Guest. J.K., et al., Structural optimization of deploying structures composed of linkages. Journal of Computing in Civil Engineering, 28(3), p. 04014010, 2012.

http://dx.doi.org/10.1061/(ASCE)CP.1943-5487.0000272

[18] Dai, L. \& Guan, F.L., Shape-sizing nested optimization of deployable structures using SQP. Journal of Central South University, 21(7), pp. 2915-2920, 2014. http://dx.doi.org/10.1007/s11771-014-2257-0

[19] Fest, E., Shea, K., Domer, B. \& Smith, I.F.C., Adjustable tensegrity structures. Journal of Structural Engineering, 129(4), pp. 515-526, 2003. http://dx.doi.org/10.1061/(ASCE)0733-9445(2003)129:4(515)

[20] Buhl, T., Jensen, F.V. \& Pellegrino, S., Shape optimization of cover plates for retractable roof structures. Computers \& Structures, 82(15), pp. 1227-1236, 2004. http://dx.doi.org/10.1016/j.compstruc.2004.02.021

[21] Ortega, N.F. \& Robles, S.I., Optimization of a telescope movable support structure by means of volumetric displacements. Structural Engineering and Mechanics, 31(4), pp. 393-405, 2009. http://dx.doi.org/10.12989/sem.2009.31.4.393

[22] Fenci, G.E., Currie, N. \& Weekes, L., Biomimetic approach for the creation of a deployable structure based on the blooming of the Morning Glory flower. In International Association for Shell and Spatial Structures (IASS) Symposium, Future Visions, Amsterdam, 2015.

[23] Rutten, D., Grasshopper: generative modeling for Rhino (Computer software)," 2007.

[24] Preisinger, C., Linking structure and parametric geometry. Architectural Design, 83(2), pp. 110-113, 2013. http://dx.doi.org/10.1002/ad.1564

[25] Autodesk Inc, Autodesk Robot Structural Analysis 2016, 2015.

[26] Thornton Tomasetti. "Introducing the Brute Force Solver," 5 August, 2016, available at: http://core.thorntontomasetti.com/introducing-the-brute-force-solver/

[27] Wolfram Research Inc., "Mathematica," Version 10.0, Wolfram Research, Inc., 2014.

[28] Fenci, G.E. \& Currie, N., Biomimetic approach for the creation of deployable canopies based on the unfolding of a beetle wing and the blooming of a flower. In Conference on Biomimetic and Biohybrid Systems, pp. 101-112, 2015.

http://dx.doi.org/10.1007/978-3-319-22979-9_11 